\title{
WISKOTT ALDRICH SYNDROME - Is transplant a better option than supportive care?
}

\author{
Nikki Agarwal ${ }^{1}$, Divyaswathi Citla Sridhar ${ }^{2}$, Sindhoosha Malay $^{3}$, Nirav Patil ${ }^{3}$, Sanjay \\ Ahuja $^{2}$, and jignesh dalal ${ }^{2}$ \\ ${ }^{1}$ MetroHealth Medical Center \\ ${ }^{2}$ UH Rainbow Babies and Children's Hospital \\ ${ }^{3}$ Case Western Reserve University
}

May 15, 2020

\begin{abstract}
Background : Wiskott Aldrich syndrome (WAS) is a rare disease and hematopoietic stem cell transplant (HCT) is considered the treatment modality of choice for WAS. Methods : We conducted a cross-sectional analysis on the Kids' inpatient database and compared hospitalization rates, complications and healthcare utilizations in the transplant and non-transplant arms. Results. : Of the 396 patients with WAS admitted between 2006-2012, 114 underwent transplant and 269 did not. The non-transplant arm included older children, female patients and more African Americans. Death rates, income and payer source were similar in both arms, however the total charge for each admission was higher in the transplant arm. ED visits were similar but elective admissions were more in the transplant arm. Length of stay was prolonged in the transplant arm. When comparing morbidities, lymphomas, ulcerative colitis and autoimmune complications of WAS were seen only in the non-transplant arm. Conclusions : Our study shows that transplant is the largest contributor to healthcare utilization in WAS patients. We noted a change in practice moving away from splenectomy in WAS patients. We identified healthcare disparities based on race and socioeconomic status and found that only a few centers across the nation manage WAS, suggesting need for wider resources for this very rare disease.
\end{abstract}

\section{INTRODUCTION :}

Wiskott Aldrich syndrome (WAS) is a rare X-linked disorder characterized by eczema, small platelets with thrombocytopenia and combined immunodeficiency with increased risk for autoimmune disorders and cancers $^{1,2}$. The incidence is 1 to 10 per million live male births, with average age at diagnosis of 24 months in families without a previously affected family member ${ }^{3}$. WAS has a wide range of phenotypes depending on the expression of the WASP gene. Therapeutic options range from supportive therapy to allogeneic bone marrow transplant and gene therapy ${ }^{4,5,6}$.

As with rare diseases, there is a paucity of randomized control trials in WAS. Observational studies have been conducted to define disease events and recognize outcomes of WAS patients treated with different therapeutic modalities. Hematopoietic stem cell transplant (HCT) as a treatment modality for WAS was first employed in $1968^{3}$. Since then, use of matched sibling donor for HCT has become a standard of care for $\mathrm{WAS}^{4,5,6}$. There are several studies showing excellent outcomes with matched sibling HCT. However, there are no studies with a direct comparison of outcomes, complications and health care utilization in patients who underwent transplant versus no transplant.

We conducted a cross sectional analysis on a large inpatient database to compare outcomes, associated 
complications and health care utilization in patients with WAS who underwent HCT versus those who received supportive care.

\section{METHODS:}

\section{(i) Study design and sample}

This was a cross-sectional analysis of pediatric hospitalizations (age $<21$ years) of patients with WAS using the Kids' Inpatient Database (KID), prepared by the Agency for Healthcare Research and Quality (AHRQ) as part of the Healthcare Resource Utilization Project (HCUP) ${ }^{7,8,9}$. The KID database is a pediatric allpayer inpatient database of pediatric discharges at children's hospitals, academic centers and community hospitals from 47 participating states. Since this study involves analysis of a publicly available de-identified data, it was exempt from Institutional Review Board review.

\section{(ii) Outcomes}

The primary outcome was to measure hospitalization among patients with WAS, who underwent HCT versus patients who received supportive care. Our secondary outcomes were to compare incidence of WAS associated complications among patients who did and did not receive HCT, assess incidence of transplant related complications among patients who underwent HCT. We also aimed to compare health care utilization among patient in both groups.

\section{(iii) Clinical variables}

Hospitalized patients with WAS were identified with anInternational Classification of Disease - ninth revision (ICD-9) code of 279.12. Among these patients, those who underwent HCT were identified using ICD-9 diagnosis code of V4281 and DRG version 24 of 481. Data collected included patient age, sex, race, death during hospitalization, length of stay in days, elective admission, number of procedures, total charges for the admission, location of the patient and primary insurance status (Medicare, Medicaid, Private Insurance and Self-pay). All ICD-9 codes listed under diagnosis were reviewed to identify complications associated with WAS and complications associated with HCT.

\section{(iv) Statistical Analysis}

HCUP-NIS sampling design and analysis have been described in previous HCUP publications ${ }^{10,11}$. We used the standard published algorithm and applied sampling weights to generate nationwide estimates. Results mentioned in this paper are weighted nationwide estimates. Data was presented as mean (standard deviation) or frequency (percentage) as appropriate. Continuous data was compared with the two-tailed independent sample t-test while categorical data was compared with the Rao-Scott adjustment for chi square test. All results are two-tailed and at the $95 \%$ confidence level. P-value of less than 0.05 was considered statistically significant for the analysis. All the analysis was performed using SAS software, version 9.4 (SAS Institute, Cary, NC). HCUP methods series recommendations were followed for estimates for standard errors and confidence intervals ${ }^{8}$.

\section{RESULTS:}

\section{Descriptive statistics (Table 1)}

We analyzed a total of 382 hospitalizations with the diagnosis of WAS. 114 had undergone HCT and 269 had not undergone HCT. Overall mean age of admission was 5 years, with admissions in transplant arm having a mean age of 3.6 years compared to 5.63 years for non-transplant arm. When subcategorized for age (Figure 1), most admissions were in the 0-5 year age in both groups, however there were more admissions in the non-transplant arm after 6 years of age compared to transplant arm $(\mathrm{p}=0.0612)$. There were 7 female patients in the non-transplant arm, the rest were all male. There were more African Americans(18.3\%) in the non- transplant arm compared to transplant arm (6.8\%). 50\% patients overall had Medicaid with equal distribution in both arms, the rest had either private insurance or not reported $(\mathrm{p}=0.395)$.

\section{Inpatient mortality}


Death rates during hospitalization were comparable in both arms (1.61\% in the non-transplant vs $1.15 \%$ in the transplant arm).

\section{Health care utilization}

Although payer source and median household income were not significantly different between the transplant and the non-transplant arms, the total charge for each admission was higher in the transplant arm. (Table 2) . ED visits were similar in both arms $(\mathrm{p}=0.56)$. Elective admissions were more in the transplant arm compared to the non-transplant arm $(\mathrm{p}=0.0244)$. Length of stay was prolonged in transplant arm (18 days) compared to non-transplant arm (6.8 days) and was statistically significant $(\mathrm{p}<0.001)$. When comparing the morbidities, lymphomas, ulcerative colitis and autoimmune complications of WAS were seen only in the non-transplant arm (Figure 2) . Of 85 centers, only $10 \%$ centers had $42 \%$ (2.8-9.1\% each) of the hospitalizations and $90 \%$ of the centers had less than $2.5 \%$ of hospitalizations each (Table 3) .

\section{DISCUSSION :}

This study is one of the largest database reviews for patients with WAS, with data from all over the United States over a 6-year period. We found very interesting results with the review of the KIDS inpatient database made available through HCUP.

The study showed 7 female patients with WAS seeking medical care, and all of them were in the nontransplant arm. Females with WASP gene mutations are usually asymptomatic as their mature blood cells have nonrandom X chromosome inactivation, with the chromosome having normal WASP gene active in most cells. Prchal et al ${ }^{12}$ looked at X-chromosome expression in the mother of two siblings with WAS and suggested that there is selection against cells expressing the defective WASP gene in the hematopoietic system in carriers. Fearon ${ }^{13}$ et al studied eight carriers of WAS defect and identified skewed patterns of X chromosome inactivation in T-cells, granulocytes and B cells. Multiple other studies have been done to look at the carrier state in $\mathrm{WAS}^{14,15,16}$. A case report for a female presenting with mild WAS symptoms was found to have $60 \%$ of normal WASP levels in peripheral blood mononuclear cells, compared to actual cases of WAS where WASP levels were $0-20 \% \cdot{ }^{17}$ She was noted to have heterozygosity with 2 distinct populations in her lymphocytes and monocytes. Many other case reports have been described with females with WAS symptoms. ${ }^{18-22}$ Hypothesis for disease expression in a female with heterozygosity to the WASP mutation are either : abnormality in transcriptional X chromosome silencing in the hematopoietic stem cells, or abnormality in the process that leads to preferential survival and expansion of cells that bear the active wild-type X chromosome. ${ }^{23}$

Post-transplant hospitalization is the largest contributor to health care utilization for transplant patients. We noted that the length of stay (LOS) was longer in transplant patients compared to the non-transplant patients, especially in admissions associated with transplant as a procedure code, with mean LOS of 8 days for non-transplant arm and 18 days in the transplant arm. In a study using national claims database of commercially insured population in the United States, Majhail et $\mathrm{al}^{24}$ showed that the median duration of hospitalization was 19 days for autologous HCT recipients and 31 days in allogenic HCT recipients. The median days of hospital stay after the transplant was 21 days in $<20$-year age group for autologous HCT and 42 days for allogenic HCT. In another multicenter cohort study ${ }^{25}$, LOS compared between different graft sources in the first 100 days after transplant for acute leukemias showed umbilical cord blood (UCB) and mismatched unrelated donor transplants (MMUD) to have a longer hospital stay compared to matched unrelated donors (MUD). The median value of LOS in the pediatric group were 50 days for single UCB, 54 days for double UCB and 60 days for MUD HCT. Our study suggests a much lesser LOS in WAS patients, but the graft source for our patients was not available in the database.

Due to restricted availability of HCT, concerns have been raised regarding disparities based on race, socioeconomic status, education and insurance status. Our study revealed more African Americans (18.3\%) in the non-transplant arm compared to transplant arm (6.8\%). This disparity could be explained by lack of donor sources, access to HCT and outcomes of HCT. Cancer facts and figures summarized by the American Cancer Society in 2019 show highest incidence of cancer and deaths in African Americans when compared 
to other races ${ }^{26}$. $\mathrm{HCT}$ is an expensive procedure, hence socioeconomic factors create a barrier to its easy access. Also, allogenic HCT needs appropriate HLA-matched donors, and the likelihood of matching between two random individuals increases if they are of the same race. The National Marrow Donor Program's Registry $^{27}$ has about 16 million potential donors, $7 \%$ of them are African Americans, $6 \%$ Asians and $1 \%$ American Indian/Alaskan Native, and they have ongoing initiatives to increase the diversity of the pool. Barker et $\mathrm{al}^{28}$ in a prospective study conducted between $2005-2017$ found that increasing registry size has not resolved the racial disparity and suggested use of alternative graft sources such as cord blood transplant. However, unavailable matched donors continue to be a barrier to HCT in these populations.

WAS is a disease identified at a young age due to multiple infections or bleeding manifestations. Sullivan et $\mathrm{al}^{29}$ found that $36 \%$ patients with WAS experience non-HCT associated deaths at a mean age of 8 years, mostly due to infections (44\%), bleeding (23\%) and malignancy (26\%). This study also found that the median survival was 20 years in patients with WAS with supportive care only. Our study showed most admissions were in the 0-5 year age group for both arms, which suggests early identification of the disease. Our hypothesis is due to increased awareness and ease of diagnosis, cases are more easily identified. Also, greater number of children $>5$ years age required admission in the non-transplant arm, suggesting increased complications and medical needs with increased age. We were further able to consolidate this hypothesis by looking at the complications in both arms. We found that none of the patients in the transplant arm developed lymphoma, whereas $5.6 \%$ patients in the non-transplant arm developed lymphoma. Malignancies in untreated WAS has been reported as $13 \%^{29}$ and $22 \%^{30}$ in two different case series, including lymphomas, leukemias and myelodysplasia. Similarly, autoimmune manifestations are much more common in untreated WAS, ranging from $22 \%$ to $72 \%$ in various case series ${ }^{29,30,31,32}$. This was similarly reflected in our study where ulcerative colitis was noted only in the non-transplant arm compared to the transplant arm. Due to limitation of our database, we were unable to compare the course of WAS in the two arms beyond 20 years of age.

Our study did not show splenectomy done in either arm. There have been controversies regarding splenectomy if a potential donor is present, but this represents a shift in practice even in the non-transplant arm. Mullen et $\mathrm{al}^{33}$ had shown median survival of 25 years in splenectomized non HCT patients, compared to less than 5 years in unsplenectomized patients. Infectious complications related to splenectomy has been shown to be significant in WAS patients receiving HCT. ${ }^{34}$ Moratto et al ${ }^{35}$ observed the long term outcomes for WAS and HCT over a 30 year period and showed that splenectomy may normalize platelet counts after HCT, however, there are associated risks of potentially fatal infections that need to be considered. Imai et al ${ }^{30}$ had similar results of improvement in platelet counts after splenectomy, but with increased risk of life-threatening meningitis/sepsis. Our study shows a significant shift in practice away from splenectomy for management of thrombocytopenia.

Our study did not show significant difference in mortality between the two arms, but it is not reliable given that ours is an inpatient database. However, our study agrees with other studies that HCTs done at $<5$-year age group have better outcomes. ${ }^{34,35}$

\section{STRENGTHS AND LIMITATIONS :}

The KID inpatient database is one of the largest pediatric inpatient databases, with each data set providing 3 -years data, with roughly 2 to 3 million discharges (unweighted), which corresponds to a national estimate of 6 to 7 million (weighted) discharges per data set. Our study has incorporated 2 datasets, which amounts to about 12-14 million (weighted) discharges and therefore, is a very powerful tool to study a rare disease like WAS.

There are several limitations to this study that need to be discussed. Since this is an administrative dataset, there are limitations to using it for secondary analysis of clinical outcomes. The data is based on each hospitalization and is patient specific, hence we are unable to provide exact rates based on patient numbers. This is an inpatient dataset, outpatient care and mortality in the outpatient setting cannot be assessed. There might be errors secondary to incorrect ICD-9 coding, which are not avoidable in a large dataset study 
like these. However, there have been several studies that have looked at the sensitivity of ICD-9 codes ${ }^{10,11}$. This dataset is a pediatric dataset for $<21$ years of age, and hence we were not able to analyze the course of the disease beyond that age.

\section{CONCLUSIONS \& FUTURE SCOPE:}

In conclusion, our study identifies that HCT is the largest contributor to healthcare utilization in WAS patients. It also identifies the healthcare disparities based on race and socioeconomic status. There is a disparity in use of splenectomy in our database compared to other studies which suggest a change in practice in this immunodeficient population. We also found that only a few centers across the nation contribute to maximum inpatient admissions for WAS, suggesting need for wider resources for this very rare disease. There is a need for a bigger national database and longitudinal studies to follow these patients and assess for long term complications.

Ethics approval : Not applicable

Consent for publication : Not applicable

Availability of data and material : The data that support the findings of this study are available from KIDS database at www.hcup-us.ahrq.gov/kidoverview.jsp. Restrictions apply to the availability of these data, which were used under license for this study. Data are available

available on request and with permission from HCUP.

Competing interests : None of the authors have any financial or non-financial competing interests to declare.

Funding : The authors have no funding to declare.

Authors' contributions : JD and SA were responsible for conceptualizing the project with NA and DS. $\mathrm{SM}$ and NP were involved in statistical analysis of the dataset. DS and NA were responsible for drafting the manuscript and review of literature. All authors read and approved the final manuscript.

Acknowledgements : To Anjali Shekar, MD for providing consent and access to the KIDS dataset.

\section{REFERENCES :}

1. Ochs HD, Filipovich AH, Veys P, Cowan MJ, Kapoor N. Wiskott Aldrich syndrome: diagnosis, clinical and laboratory manifestations, and treatment. Biol Blood Marrow Transplant 2009;15 Suppl 1:84-90.

2. Massaad MJ, Ramesh N, Geha RS. Wiskott-Aldrich syndrome: a comprehensive review. Ann N Y Acad Sci 2013;1285:26-43.

3. Buchbinder D, Nugent DJ, Fillipovich AH. Wiskott-Aldrich syndrome: Diagnosis, current management, and emerging treatments. Appl Clin Genet 2014;7:55-66.

4. Parkman R et al. Complete correction of the Wiskott-Aldrich Syndrome by allogeneic bone-marrow transplantation. N Engl J Med 1978;298:921-927.

5. Filipovich $\mathrm{AH}$ et al. Impact of donor type on outcome of bone marrow transplantation for WiskottAldrich syndrome: collaborative study of the International Bone Marrow Transplant Registry and the National Marrow Donor Program. Blood 2001;97:1598-1603.

6. Shin CR et al. Outcomes following hematopoietic cell transplantation for Wiskott-Aldrich syndrome. Bone Marrow Transplantation 2012;47:1428-1435.

7. HCUP Kids' Inpatient Database (KID). Healthcare Cost and Utilization Project (HCUP). 2006 and 2009. Agency for Healthcare Research and Quality, Rockville, MD. www.hcupus.ahrq.gov/kidoverview.jsp.

8. Houchens R. Inferences with HCUP State Databases Final Report. HCUP Methods Series Report. U.S. Agency for Healthcare Research and Quality, 2010. https://www.hcupus.ahrq.gov/reports/methods/2010_05.pdf 
9. HCUP Quality Control Procedures. Rockville, MD: Agency for Healthcare Research and Quality. https://www.hcup-us.ahrq.gov/db/quality.jsp

10. Steiner C, Elixhauser A, Schnaier J. The healthcare cost and utilization project: an overview. Eff Clin Pract 2002;5:143-151.

11. Heckbert SR et al. Comparison of self-report, hospital discharge codes, and adjudication of cardiovascular events in the Women's Health Initiative. Am J Epidemiol 2004;160:1152-1158.

12. Prchal JT et al. Wiskott-Aldrich syndrome : cellular impairments and their implication for cellular detection. Blood 1980;56:1048-1054.

13. Fearson ER, Kohn DB, Winkelstein JA, Vogelstein B, Blaese RM. Carrier detection in the Wiskott Aldrich syndrome. Blood 1988;72:1735-1739.

14. Greer WL, Kwong PC, Peacocke M, Ip R, Rubin LA, Siminovitch KA. X-chromosome inactivation in Wiskott-Aldrich syndrome: a marker for detection of the carrier state and identification of cell lineages expressing gene defect. Genomics 1989;4:60-67.

15. Mantuano $\mathrm{E}$ et al. Analysis of $\mathrm{X}$-chromosome inactivation in bone marrow precursors from carriers of Wiskott-Aldrich syndrome and X-linked severe combined immunodeficiency : evidence the WiskottAldrich gene is expressed prior to granulocyte-macrophage colony-forming unit. Immunodeficiency 1993;4:271-276.

16. Wengler G, Gorlin JB, Williamson JM, Rosen FS, Bing DH. Nonrandom inactivation of the X chromosome in early lineage hematopoietic cells in carriers of Wiskott Aldrich syndrome. Blood 1995;85:24712477 .

17. Lutskiy MI, Sasahara Y, Kenney DM, Rosen FS, Remold-O'Donnell E. Wiskott-Aldrich syndrome in a female. Blood 2002;100(8):2763-2768.

18. Tornai I, Kiss A, Laczko J. Wiskott-Aldrich syndrome in a heterozygous carrier woman. Eur J Haematol 1989;42:501-502.

19. Lin CY, Hsu HC. Acute immune complex mediated glomerulonephritis in a Chinese girl with Wiskott Aldrich syndrome variant. Ann Allergy 1984;53:74-78.

20. Russell SJ, Nisen PD. Random X chromosome inactivation in a female with a variant of Wiskott Aldrich syndrome. Br J Haematol 1995;90:210-212.

21. Conley ME, Wang WC, Parolini O, Shapiro DN, Campana D, Siminovitch KA. Atypical WiskottAldrich syndome in a girl. Blood 1992;80:1264-1269.

22. Parolini O et al. X-linked Wiskott-Aldrich syndrome in a girl. N Engl J Med 1998;338:291-295.

23. Puck JM, Willard HF. X inactivation in females with X linked disease. N Engl J Med. 1998;338:325328.

24. Majhail NS, Mau LW, Denzen EM, Arneson TJ. Costs of autologous and allogeneic hematopoietic cell transplantation in the United States: a study using a large national private claims database. Bone Marrow Transplant 2013;48(2):294-300.

25. Ballen KK et al. Hospital length of stay in the first 100 days after allogeneic hematopoietic cell transplantation for acute leukemia in remission: comparison among alternative graft sources. Biol Blood Marrow Transplant 2014;20(11):1819-1827.

26. Cancer Facts \& Figures 2019. Atlanta, American Cancer Society (2019). https://www.cancer.org/research/cancer-facts-statistics/all-cancer-facts-figures/cancer-facts-figures2019.html

27. National Marrow Donor Program Registry Be A Match Statistics (2016). https://bethematch.org/about-us/financial-information/annual-report/

28. Barker JN et al. Racial disparities in access to HLA-matched unrelated donor transplants: a prospective 1312-patient analysis. Blood Adv 2019;3(7):939-944.

29. Sullivan KE, Mullen CA, Blease RM, Winkelstein JA. A multinational survey of the Wiskott-Aldrich syndrome. J Pediatrics 1994;125(6):876-885.

30. Imai K. et al. Clinical course of patients with WASP gene mutations. Blood 2004;103(2):456-464.

31. Dupuis-Girod S et al. Autoimmunity in Wiskott-Aldrich Syndrome: Risk factors, Clinical features and outcome in a single center cohort of 55 patients. Pediatrics 2003;111(5):e622-e627. 
32. Schurman S, Candotti F. Autoimmunity in Wiskott-Aldrich syndrome. Current Opinion in Rheumatology 2003;15(4):446-453.

33. Mullen CA, Anderson KD, Blease RM. Splenectomy and/or bone marrow transplantation in management of the Wiskott-Aldrich syndrome: long term follow-up of 62 cases. Blood 1993;82(10):2961-2966.

34. Ozsahin H. et al. Long-term outcome following hematopoietic stem-cell transplantation in WiskottAldrich syndrome: collaborative study of the European Society for Immunodeficiencies and European Group for Blood and Marrow Transplantation. Blood 2008;111(1):439-445.

35. Moratto D et al. Long-term outcome and lineage-specific chimerism in 194 patients with WiskottAldrich syndrome treated by hematopoietic cell transplantation in the period 1980-2009: an international collaborative study. Blood 2011;118(6):1675-168

\section{Hosted file}

Tables for WAS manuscript.docx available at https://authorea.com/users/322231/articles/ 451308-wiskott-aldrich-syndrome-is-transplant-a-better-option-than-supportive-care 
Figure 1: Age distribution - No HCT vs HCT

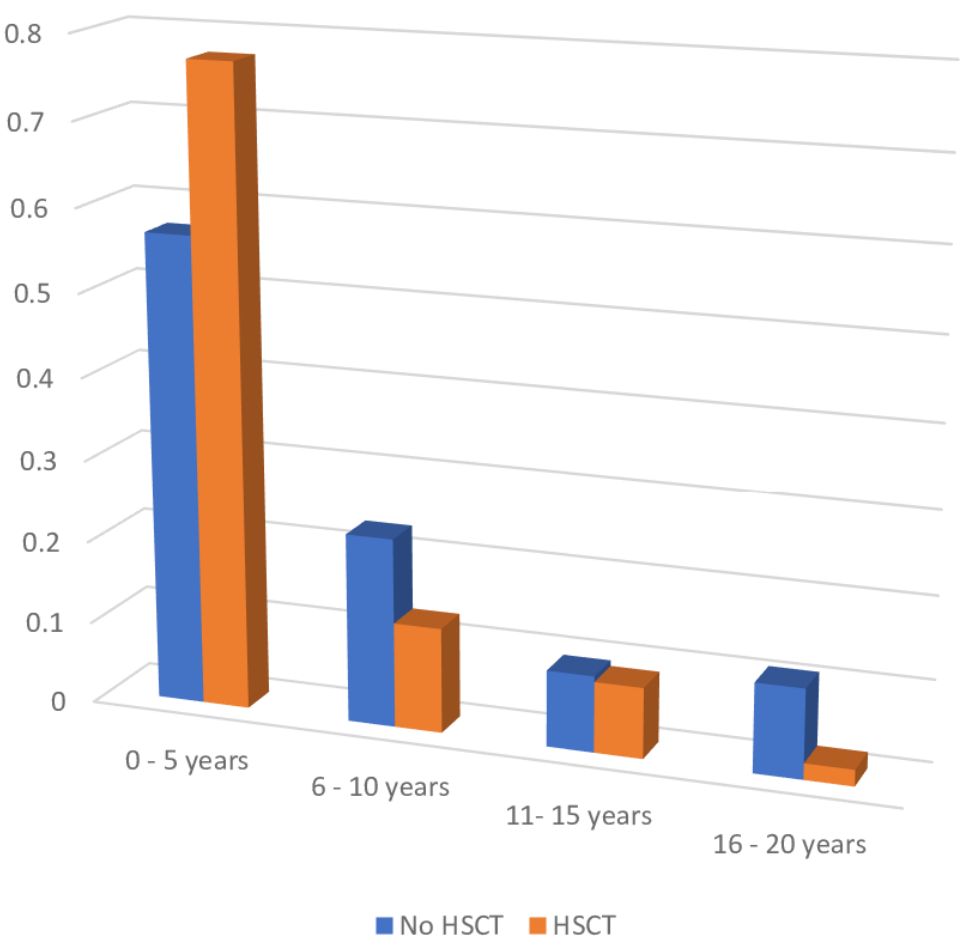

Figure 2. Co-morbidities

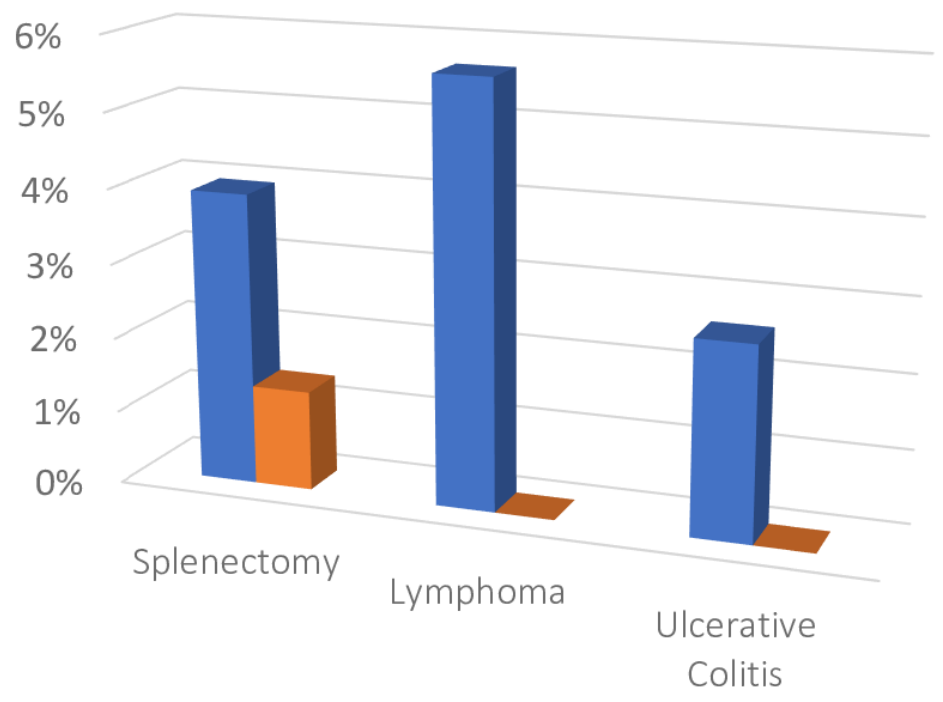

- No HSCT $\quad$ HSCT 